\title{
COMPORTAMIENTO AGRONÓMICO DE POBLACIONES F 2 DE HÍBRIDOS DE TOMATE (Solanum lycopersicum L.)
}

\section{AGRONOMIC PERFORMANCE OF F POPULATIONS FROM TOMATO HYBRIDS (Solanum lycopersicum L.)}

\author{
Enrique Hernández-Leal ${ }^{1}$, Ricardo Lobato-Ortiz ${ }^{1 \star}$, J. Jesús García-Zavala ${ }^{1}$, Delfino Reyes-López ${ }^{2}$, \\ Alonso Méndez-López ${ }^{3}$, Olga Bonilla-Barrientos ${ }^{1}$ y Aurelio Hernández-Bautista ${ }^{1}$
}

\begin{abstract}
${ }^{1}$ Postgrado en Recursos Genéticos y Productividad-Genética, Colegio de Postgraduados, Campus Montecillo. Km. 36.5 carr. México-Texcoco. 56230, Montecillo, Texcoco, Edo. de México. ${ }^{2}$ Facultad de Ingeniería Agrohidráulica, Benemérita Universidad Autónoma de Puebla. San Juan Acateno, Teziutlán, Puebla. ${ }^{3}$ Universidad Autónoma Chapingo. Km. 38.5 carr. México-Texcoco. 56230, Chapingo, Edo. de México. Tel: 01 (595) 952-1500.

*Autor para correspondencia (rlobato@colpos.mx)
\end{abstract}

\section{RESUMEN}

Casi toda la semilla de tomate (Solanum lycopersicum L.) que se utiliza para siembras comerciales en México proviene de empresas extranjeras, por lo que el costo de dicha semilla es muy alto. Esta situación provoca que en ocasiones el pequeño productor siembre semilla de generaciones tempranas $\left(\mathrm{F}_{2}\right.$ y $\left.\mathrm{F}_{3}\right)$ de híbridos, con la posible pérdida de rendimiento y calidad de fruto. En este trabajo se evaluó el rendimiento de fruto por planta (PTF), componentes de rendimiento y algunos caracteres de calidad de siete híbridos de tomate tipo Saladette de crecimiento indeterminado, y sus respectivas generaciones $F_{2}$, bajo un diseño experimental de bloques completos al azar con cuatro repeticiones, en plantas crecidas en condiciones de invernadero e hidroponía. El análisis de varianza de contrastes de los promedios $F_{1}$ $v s$. $\mathrm{F}_{2}$ detectó diferencias significativas para la variable $\mathrm{PTF}$ en cinco de los siete genotipos ('Sun 7705, 'Moctezuma', 'Cuauhtémoc', 'Reserva' y 'Cid'). En las variedades 'Espartaco' y 'Loreto' las diferencias no fueron significativas, es decir, la reducción del rendimiento en las generaciones $\mathrm{F}_{2}$ fue mínima, lo cual sugiere que la depresión endogámica de una generación $\mathrm{F}_{2}$ depende particularmente de la constitución genética de las líneas que hayan dado lugar al híbrido comercial. Para las variables número total de frutos, contenido de sólidos solubles totales, firmeza y longitud de fruto $\mathrm{y} \mathrm{pH}$ del jugo del fruto, de manera general y para la mayoría de los genotipos se observó que no hubo diferencias estadísticas entre los promedios de las generaciones $F_{1}$ y $F_{2}$, lo cual indica que la magnitud de estas características, algunas de ellas de calidad, se mantiene en individuos segregantes $\mathrm{F}_{2}$.

Palabras clave: Solanum lycopersicum, germoplasma, mejoramiento genético.

\section{SUMMARY}

Most tomato (Solanum lycopersicum L.) seed used in commercial in Mexico crops are acquired from foreign companies; this seed is very expensive. To avoid the expense, smaller farmers try sowing hybrid seeds from early generations $\left(\mathrm{F}_{2}\right.$ and $\left.\mathrm{F}_{3}\right)$, possibly losing fruit yield and fruit quality. This work evaluated fruit yield per plant (PTF), yield components and some quality related traits of seven Saladette tomato hybrids of indeterminate growth and their respective $\mathrm{F}_{2}$ generations. A ran domized completely block design with four replications was employed in plants grown under greenhouse and hydroponic conditions. The analysis of variance of contrasts of averages between $\mathrm{F}_{1} v s . \mathrm{F}_{2}$ detected significant differences for the variable PTF in five out of the seven genotypes ('Sun 7705', 'Moctezuma,' 'Cuauhtémoc', 'Reserva' y 'Cid'). In the case of the other two genotypes ('Loreto' and 'Espartaco'), the differences were not significant, thus indicating that the reduction of fruit yield in $F_{2}$ generations was minimal. These results suggest that inbreeding depression in an $\mathrm{F}_{2}$ generation depends particularly on the genetic constitution of the lines that formed the commercial hybrid. For total fruit number, total soluble solids content, fruit firmness, length and $\mathrm{pH}$, in a general way and for the majority of the genotypes, there were no statistical differences between the averages of the $F_{1}$ and $F_{2}$ generations. These findings show that the magnitude of these characteristics remained without change in $F_{2}$ segregating individuals, some of them being quality related traits.

Index words: Solanum lycopersicum, germplasm, genetic improvement.

\section{INTRODUCCIÓN}

El tomate (Solanum lycopersicum L.) es la hortaliza más popular y aceptada en la cultura gastronómica del mundo, y se cultiva en más de cien países para consumo en fresco o para su industrialización. Los diez principales países productores que concentran más de $80 \%$ del total mundial son: China, Estados Unidos, India, Egipto, Turquía, Italia, Irán, España, Brasil y México. Los tres primeros marcan las tendencias de precios y consumo mundiales (FAO, 2012).

En México se siembran anualmente alrededor de 80000 ha, con un rendimiento promedio de $28.7 \mathrm{t} \mathrm{ha}^{-1}$ en condiciones de campo, por lo cual es la hortaliza más importante por la superficie sembrada, su volumen en el mercado nacional, la generación de empleos y su valor de producción (Nieto y Velasco, 2006). Además, como en casi todos los países consumidores, la calidad del tomate fresco (sabor, color, tamaño, etc.) se sobrepone al precio y valor nutritivo al momento de la elección de compra por parte del consumidor final (González et al., 2004).

El tomate ha sido y es de gran importancia socioeconómica para México, ya que anualmente se exporta a Estados Unidos 90 \% de la producción (González y Martínez, 2002), principalmente en la temporada invernal (De Giglio, 2003). 
El mejoramiento genético en tomate se ha orientado al rendimiento, adaptación a condiciones cálidas y húmedas, resistencia a enfermedades y calidad del fruto (Bai y Lindhout, 2007; Scott, 2008), esta última medida por contenido de sólidos solubles totales (SST), color del fruto, acidez titulable (AT) y pH del jugo (Stevens, 1973).

Debido a las dificultades relacionadas con la selección fenotípica para obtener un mayor rendimiento, mediante marcadores moleculares se han identificado regiones genómicas que están directa o indirectamente asociadas con el rendimiento (Foolad, 2007). Además, las nuevas técnicas de producción, las recientes exigencias agrícolas e industriales, y los continuos problemas derivados de nuevas enfermedades y plagas, han obligado a desarrollar nuevas variedades de manera continua (Escalona et al., 2009).

El mejoramiento genético de tomate en el mundo es desarrollado principalmente por universidades extranjeras y empresas transnacionales (Scott, 2008), las cuales venden semillas híbridas que se cotizan en dólares o euros. Debido a esto, los costos de producción son considerablemente altos para muchos productores mexicanos de tomate, pues los precios frecuentemente rebasan los 0.50 centavos de dólar por cada semilla híbrida (Juárez et al., 2000).

Por esta razón, la mayoría de pequeños agricultores utiliza semilla de las generaciones $\mathrm{F}_{2} \mathrm{o}_{3}$ derivada de híbridos comerciales para reducir costos, bajo el supuesto de que el rendimiento y calidad de fruto de ambas generaciones filiales es similar a la de la $\mathrm{F}_{1}$. Por ser la $\mathrm{F}_{2}$ una generación segregante, cuando el agricultor siembra esa semilla las plantas resultantes son diferentes unas de otras, lo que implica inconvenientes, como reducción drástica en la productividad, merma en la resistencia a insectos y enfermedades, maduración desuniforme, y menor calidad industrial, entre otras (De Miranda y Anderson, 2001).

De acuerdo con Poehlman y Allen (2003), en especies autógamas la segregación en la $\mathrm{F}_{2}$ del híbrido provoca reducción del rendimiento y de características de calidad del fruto, debido a que el grado de heterocigosis se reduce a la mitad, así como al efecto de la endogamia (Jarne y Charlesworth, 1993) conocida como depresión endogámica.

En cambio, otros investigadores consideran que la heterosis en especies autógamas como el tomate, no se expresa en igual magnitud que en especies alógamas (Foolad, 2007). Aun así, existe evidencia experimental de segregación transgresiva en la generación $\mathrm{F}_{2}$ en unos genotipos de tomate (De Vicente y Tanksley, 1993; Pohelman y Allen, 2003; Shivaprasad et al., 2012), lo que permite que en ocasiones aparezcan plantas con frutos de mayor peso (Mohamed, 1998).
Al respecto, se ha demostrado que la segregación transgresiva es muy común a través de especies, como en una investigación donde $44 \%$ de 1229 características examinadas fueron transgresivas (Rieseberg et al., 1999). Por otra parte, la depresión endogámica en especies autógamas es relativamente pequeña en comparación con la que se esperaría en especies alógamas (Charlesworth y Charlesworth, 1987), debido a que en las primeras el proceso de autofecundación recurrente tiene un efecto depurador de alelos recesivos deletéreos.

El objetivo de este trabajo fue comparar el rendimiento de fruto y otras características agronómicas de híbridos comerciales $\mathrm{F}_{1}$ de tomate tipo Saladette con respecto a su $\mathrm{F}_{2}$, bajo la hipótesis de que el comportamiento agronómico de la generación $\mathrm{F}_{2}$ no difiere significativamente del de la $\mathrm{F}_{1}$.

\section{MATERIALES Y MÉTODOS}

\section{Material genético}

Se evaluaron siete híbridos comerciales de tomate tipo Saladette de crecimiento indeterminado ('Moctezuma', 'Cuauhtémoc', 'Espartaco' y 'Cid', los cuatro de la empresa Harris Morgan; 'Sun 7705', de Nunhems; 'Loreto', de Séminis; y 'Reserva', de Vilmorin), y sus respectivas generaciones $\mathrm{F}_{2}$. La semilla $\mathrm{F}_{2}$ se obtuvo en condiciones de invernadero e hidroponía durante el ciclo primavera-verano 2011 en Montecillo, Estado de México, cuya localización geográfica es $19^{\circ} 30^{\prime} \mathrm{LN}$ y $98^{\circ} 53^{\prime} \mathrm{LO}$, a una altitud de $2250 \mathrm{~m}$, donde se sembraron diez plántulas por híbrido. La siembra fue el 22 de marzo y el trasplante el 23 de abril en bolsas de polietileno $(40 \times 40 \mathrm{~cm})$ rellenas con tezontle rojo como sustrato. $\mathrm{Al}$ momento de la cosecha, se seleccionaron las mejores tres plantas de cada genotipo, con base en el porte y sanidad de las mismas, tamaño y forma de fruto, número de racimos de fruto y número de frutos por racimo. Se hicieron tres cosechas (a los 82, 94 y 136 días después del trasplante, ddt). Con las semillas de la primera cosecha de todos los frutos de las tres plantas seleccionadas por genotipo, se formó un compuesto mecánico balanceado.

\section{Diseño experimental}

Los catorce genotipos, siete híbridos $\mathrm{F}_{1} \mathrm{y}$ sus respectivas generaciones $\mathrm{F}_{2}$, se evaluaron bajo un diseño experimental de bloques completos al azar, con cuatro repeticiones y cinco individuos por repetición, con un total de 56 unidades experimentales. La unidad experimental consistió de cinco plantas.

La siembra de semilla se hizo en agosto de 2011, el trasplante a los 52 días después de la siembra (dds), y la cosecha en dos cortes, uno en enero y otro en febrero de 2012. Las 
plantas se manejaron a un solo tallo y se tutoraron con hilo de rafia.

\section{Variables medidas}

Se registró información del número total de frutos por planta (NTF), peso total de frutos por planta (PTF, en g), el peso promedio de fruto (PPF, en g), diámetro (DF, en $\mathrm{mm}$ ) y longitud de fruto (LF, en $\mathrm{mm}$ ), contenido de sólidos solubles totales (SST, en ${ }^{\circ} \mathrm{Bx}$ ), acidez/pH $(\mathrm{pH})$ y firmeza del fruto (FIR, en newtons -N-). NTF y PTF se calcularon con base en el promedio de las cinco plantas por repetición, cosechadas individualmente y sumando los dos primeros cortes. Los valores de las demás variables corresponden a los promedios de una muestra de cinco frutos de cada una de las cinco plantas por repetición, medidas en el primer corte.

\section{Análisis estadístico}

Se aplicó un análisis de varianza a cada variable, de acuerdo con el diseño experimental de bloques completos al azar, mediante el modelo lineal general (PROC GLM) de SAS V9 (SAS Institute, 2002). Para comparar los promedios de los híbridos $\mathrm{F}_{1}$ vs. sus respectivas generaciones $\mathrm{F}_{2}$, se utilizó el procedimiento GLM y CONTRAST, así como la prueba de Tukey $(\mathrm{P}=0.05)$, también con SAS V9.

La depresión endogámica (DEP, en \%) se estimó con los promedios de las diferencias $\left(\mathrm{F}_{1}-\mathrm{F}_{2}\right) / \mathrm{F}_{1}$, expresadas en porcentaje, y multiplicada por (-1) para indicar una disminución de la magnitud de la variable a evaluar.

\section{Manejo del cultivo}

Se aplicó la solución nutritiva (Steiner, 1984) en cantidades que se modificaron según las etapas fenológicas de los genotipos. Se aplicaron cuatro fertirriegos con la solución de Steiner durante el ciclo, en los que solamente se modifi- có el gasto diario por planta; del trasplante hasta floración el gasto fue de $0.5 \mathrm{~L} \mathrm{~d}^{-1}$ por planta, y de floración hasta fructificación el gasto fue de $1.5 \mathrm{~L} \mathrm{~d}^{-1}$ por planta. El control de plagas se hizo con aplicaciones periódicas de Confidor ${ }^{\circledR}$ (i.a. imidacloprid) a una dosis de $0.5 \mathrm{~mL} \mathrm{~L}^{-1}$. Para prevenir el tizón tardío (Phytophthora infestans Mont. de Bary) se hizo una aplicación mensual preventiva con Ridomil Gold ${ }^{\circ}$ a $250 \mathrm{~g} / 100 \mathrm{~L}$ de agua (i. a. metalaxil M + clorotalonil).

\section{RESULTADOS Y DISCUSIÓN}

En las variedades 'Sun 7705, 'Moctezuma,' 'Cuauhtémoc' y 'Cid' hubo tres variables en común (PTF, PPF y DF), con diferencias significativas entre la $\mathrm{F}_{1}$ y la $\mathrm{F}_{2}$, seguidas de 'Espartaco' con dos variables (PPF y DF), 'Reserva' con una (PTF), y 'Loreto' en la que no hubo diferencias significativas entre ambas generaciones filiales para variable alguna (Cuadro 1). Esto implica que el grado de depresión endogámica varía según el híbrido de que se trate y de la variable que se utilice, como se ilustra en la Figura 1.

Los resultados aquí obtenidos en cuanto al peso por fruto (PPF) coinciden con los reportados por Martínez et al. (2005), quienes tampoco encontraron diferencias significativas entre las generaciones $\mathrm{F}_{1}$ y $\mathrm{F}_{2}$ en 18 híbridos de hábito determinado. El PPF es una variable importante porque está estrechamente relacionada con la producción total por planta (Bartkaite, 2001).

En todas las variedades, el peso total de frutos por planta fue mayor en la $\mathrm{F}_{1}$ que en la $\mathrm{F}_{2}$, con reducciones porcentuales entre 17.5 y $66.1 \%$ (Cuadro 2). Con excepción de 'Loreto' y 'Espartaco', en las demás variedades tales diferencias fueron significativas. La superioridad de $\mathrm{F}_{1}$ respecto a $\mathrm{F}_{2}$ también fue general en todas las variedades para el peso promedio del fruto (con reducciones de 11.8 a $36.1 \%$, significativas solamente para 'Sun 7705 ,

Cuadro 1. Cuadrados medios de los contrastes entre las generaciones $\mathrm{F}_{1}$ vs. $\mathrm{F}_{2}$ de siete variedades de tomate tipo Saladette.

\begin{tabular}{|c|c|c|c|c|c|c|c|c|}
\hline Genotipos & PTF & NTF & PPF & SST & FIR & LF & DF & $\mathrm{pH}$ \\
\hline 'Sun 7705 ' vs. Sun $7705-\mathrm{F}_{2}$ & $987062^{* *}$ & 21 & $1390^{*}$ & 0.3961 & 0.0561 & 3 & $72^{*}$ & 0.0041 \\
\hline 'Loreto' vs. Loreto- $\mathrm{F}_{2}$ & 35951 & 3 & 367 & 0.03 & 0.0006 & 0.022 & 25 & 0.0253 \\
\hline 'Moctezuma' vs. Moctezuma- $\mathrm{F}_{2}$ & $942063^{\star *}$ & 32 & $3556^{*}$ & 0.3655 & 0.0041 & $348.3^{* *}$ & $101^{\star *}$ & 0.0091 \\
\hline 'Cuauhtémoc' vs. Cuauhtémoc- $\mathrm{F}_{2}$ & $368370^{*}$ & 3 & 1047 & 0.0162 & 0.0666 & 95 & $56^{*}$ & 0.0378 \\
\hline 'Reserva’ vs. Reserva- $\mathrm{F}_{2}$ & $437416^{\star}$ & 18 & 396 & 0.0968 & 0.0595 & 11.8 & 25 & 0.0253 \\
\hline 'Espartaco’ vs. Espartaco- $\mathrm{F}_{2}$ & 140204 & 6 & 1162 & 0.0406 & 0.177 & 120 & $46^{*}$ & 0.005 \\
\hline ‘Cid’ vs. Cid-F ${ }_{2}$ & $295596^{*}$ & 0 & $3465^{\star}$ & 0.2813 & $0.6786^{*}$ & 100 & $114^{\star \star}$ & 0.0365 \\
\hline CV (\%) & 32.9 & 31.1 & 11.8 & 7.1 & 22.3 & 7.3 & 5.6 & 8.5 \\
\hline
\end{tabular}

*, ** Significativo a $\mathrm{P} \leq 0.05$ y a $\mathrm{P} \leq 0.01$, respectivamente. $\mathrm{PTF}=$ peso total de frutos por planta $(\mathrm{g}) ; \mathrm{NTF}=$ número total de frutos por planta; $\mathrm{PPF}=$ peso individual del fruto $(\mathrm{g}) ; \mathrm{SST}=$ contenido de sólidos solubles totales $\left({ }^{\circ} \mathrm{Brix}\right) ; \mathrm{FIR}=$ firmeza $(\mathrm{N})$ del fruto; LF = longitud de fruto $(\mathrm{mm}) ; \mathrm{DF}=$ diámetro de fruto $(\mathrm{mm})$. 

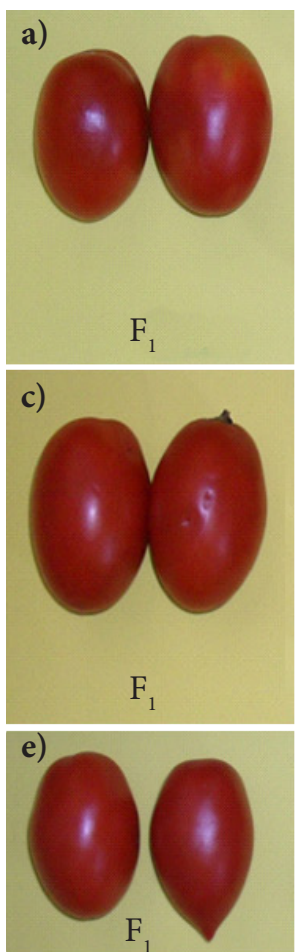
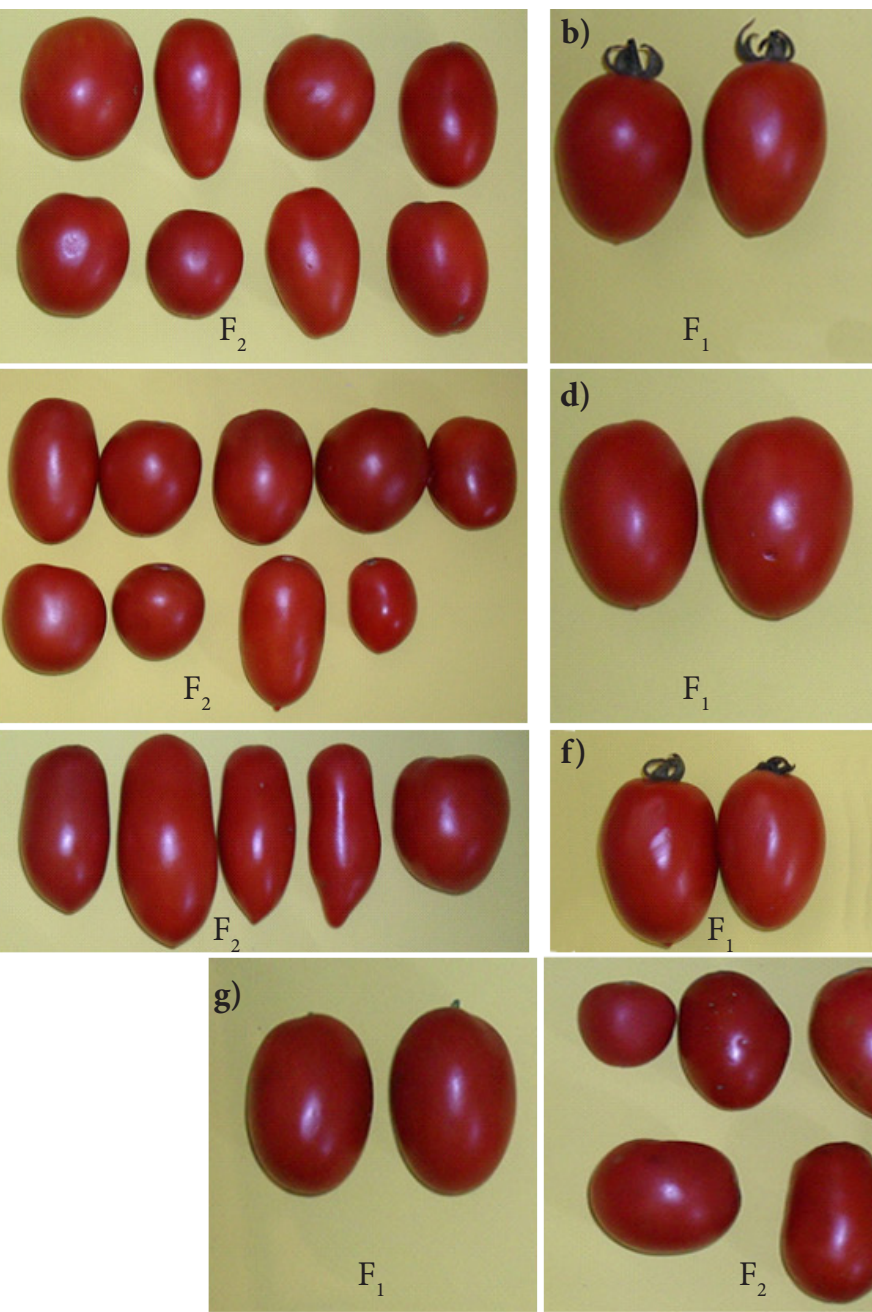
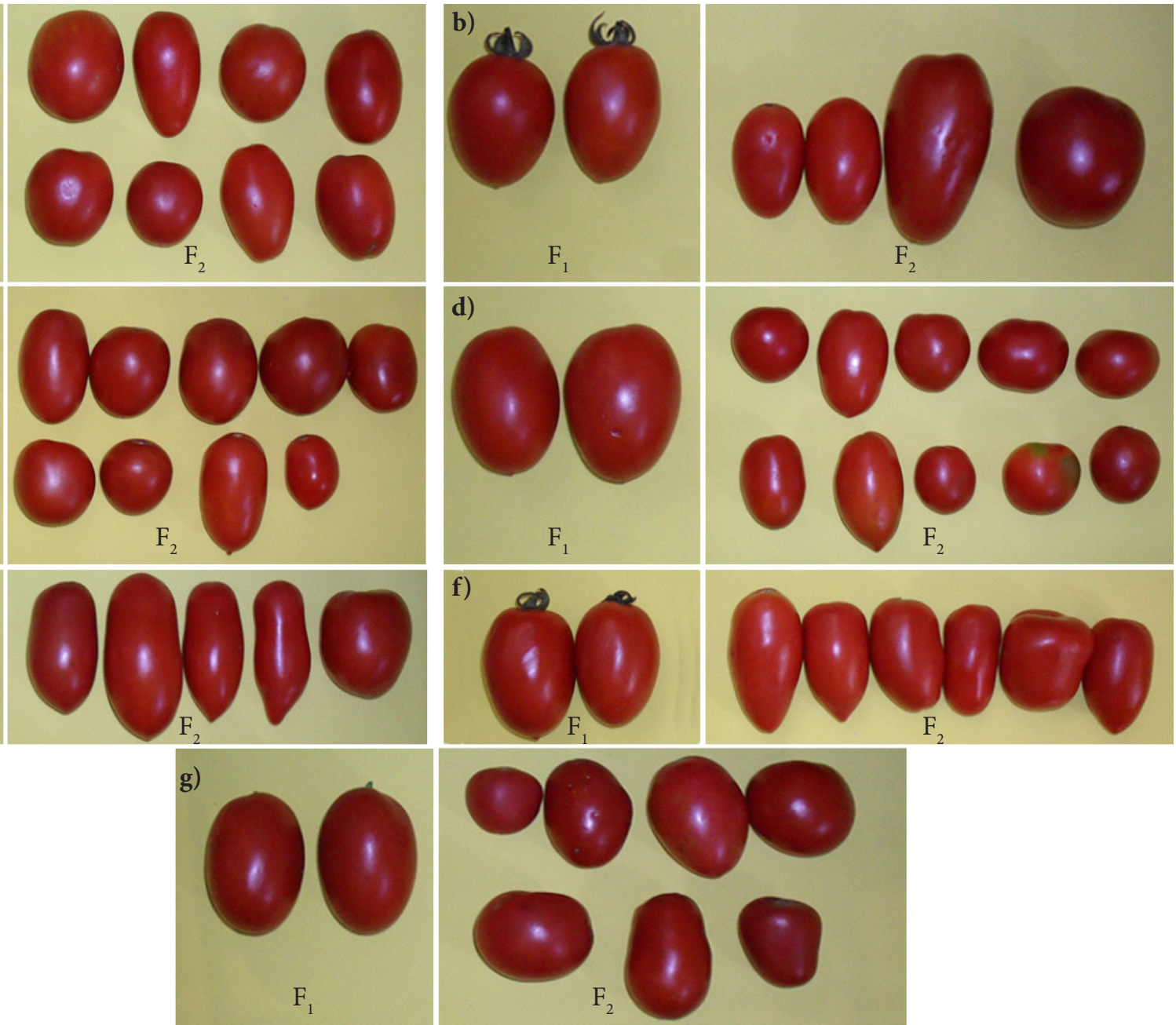

Figura 1. Variación en frutos de la generación $F_{2}$ con relación a la $F_{1}$, en siete variedades de tomate: a) 'Loreto'; b) 'Moctezuma'; c) 'Cuauhtémoc'; d) 'Reserva'; e) 'Espartaco'; f)'Sun 7705'; y g) 'cid'.

'Moctezuma' y 'Cid'), y para el diámetro del fruto, donde la depresión endogámica varió de 6.5 a $14.2 \%$ y que fue significativa para todas las variedades, excepto para 'Loreto' y 'Reserva').

El híbrido 'Moctezuma' fue el genotipo que presentó mayor abatimiento del rendimiento de fruto y de sus componentes en la generación $\mathrm{F}_{2}$ con respecto a su $\mathrm{F}_{1}$, del orden de $65 \%$ en peso total de frutos, $36.4 \%$ en número total de frutos, $36.1 \%$ en peso promedio del fruto individual, 17.3 $\%$ en longitud, y $13.7 \%$ en diámetro (Cuadro 2).

En cambio, en el híbrido 'Loreto' las características de los frutos no fueron estadísticamente diferentes entre $\mathrm{F}_{1}$ y $\mathrm{F}_{2}$ (Cuadro 2), y el abatimiento de la $\mathrm{F}_{2}$ respecto a la $\mathrm{F}_{1}$ fue equivalente a solo $17.5 \%$ para peso total, $11.8 \%$ para peso individual y $6.5 \%$ para diámetro del fruto (Cuadro 2). Destaca que el híbrido 'Moctezuma' fue el de mayor depresión endogámica en PTF (1056 vs. $369 \mathrm{~g} /$ planta) y que el híbrido
'Loreto' presentó la menor depresión (766 vs. $632 \mathrm{~g} /$ planta), por lo que la $\mathrm{F}_{2}$ de este híbrido sería una buena opción de siembra para productores de tomate en baja escala.

De acuerdo con los resultados del análisis de contrastes, no hubo diferencias estadísticas $(\mathrm{P} \leq 0.05)$ entre las generaciones $\mathrm{F}_{1}$ y $\mathrm{F}_{2}$ de todas las variedades, para las variables número total de frutos (NTF), contenido de sólidos solubles totales (SST), firmeza del fruto (FIR) excepto en 'Cid', longitud de fruto (LF) excepto la variedad 'Moctezuma', y $\mathrm{pH}$, lo cual indica que estas características, algunas de ellas de calidad, se mantienen en la generación segregante $\mathrm{F}_{2}$, por lo que los pequeños productores de tomate podrían usar la semilla $\mathrm{F}_{2}$ para siembras comerciales, sin que los frutos demeriten en esos criterios de calidad.

En este contexto, el costo de semilla podría reducirse ya que este rubro resulta oneroso para muchos productores mexicanos de tomate que utilizan básicamente híbridos, 
Cuadro 2. Comparación de medias y reducción porcentual entre híbridos de tomate tipo Saladette de crecimiento indeterminado y sus $\mathrm{F}_{2}$. Texcoco, México.

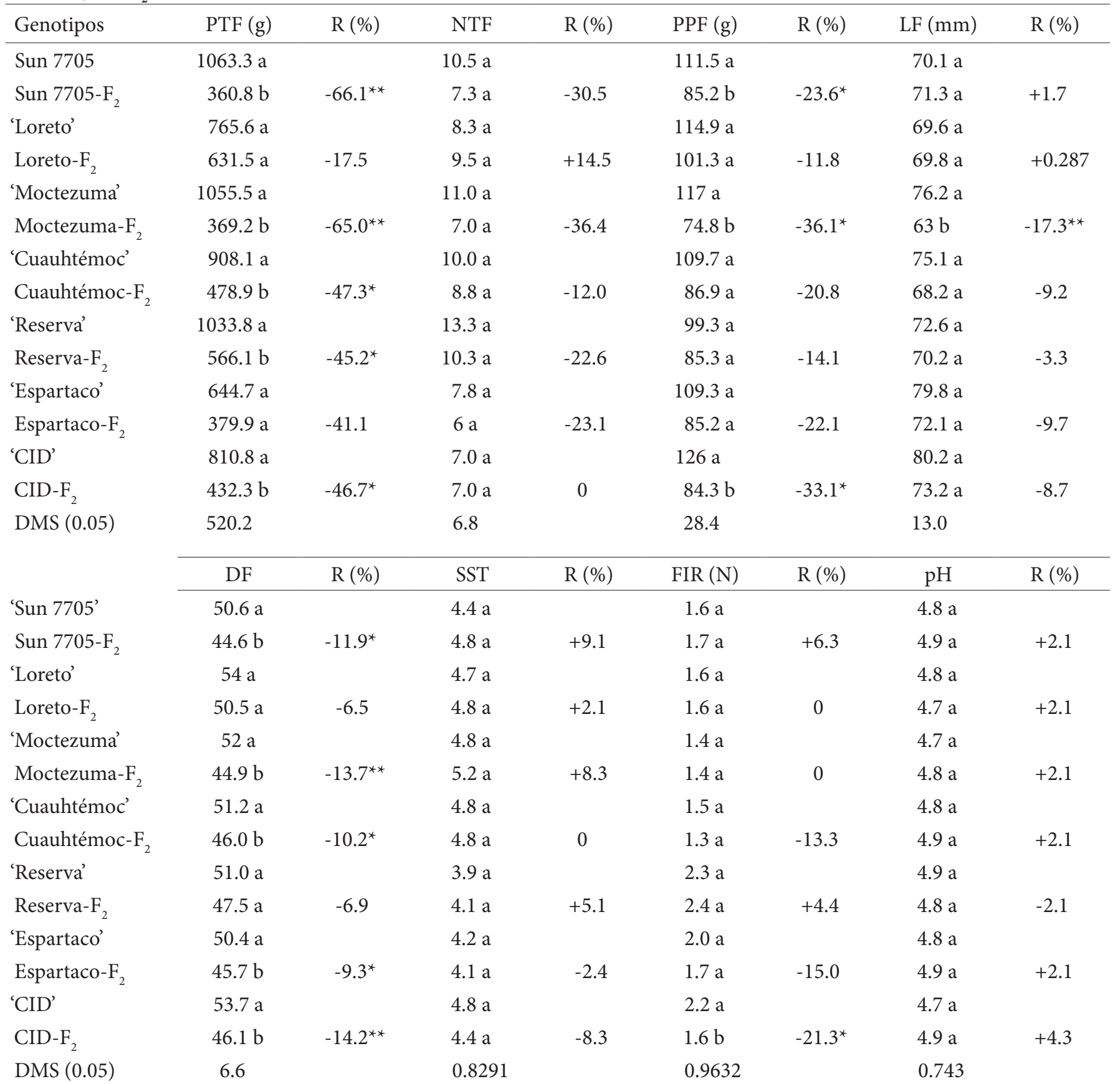

*, ** Significativo a $\mathrm{P} \leq 0.05)$ y a $\mathrm{P} \leq 0.01$, respectivamente. Medias con la misma letra en columna entre tratamientos, son estadísticamente iguales $(\alpha=0.05) ; \mathrm{R}=$ reducciones porcentuales significativas de acuerdo con la prueba de contrastes del Cuadro $1 . \mathrm{PTF}=$ peso total de frutos por planta $(\mathrm{g})$; NTF $=$ número total de frutos por planta; $\mathrm{PPF}=$ peso individual del fruto $(\mathrm{g}) ; \mathrm{LF}=$ longitud de fruto $(\mathrm{mm}) ; \mathrm{DF}=$ diámetro de fruto $(\mathrm{mm})$; SST = contenido de sólidos solubles totales $\left({ }^{0} \mathrm{Brix}\right) ; \mathrm{FIR}=$ firmeza $(\mathrm{N})$ del fruto; $\mathrm{pH}$ = potencial de iones hidrógeno. 
Cuadro 3. Errores estándar de las medias de híbridos de tomate tipo Saladette y sus respectivas $\mathrm{F}_{2}$. Texcoco, México.

\begin{tabular}{|c|c|c|c|c|c|c|c|c|}
\hline Genotipos & $\mathrm{PTF}(\mathrm{g})$ & NTF & $\mathrm{PPF}(\mathrm{g})$ & $\mathrm{LF}(\mathrm{mm})$ & $\mathrm{DF}(\mathrm{mm})$ & SST & $\operatorname{FIR}(\mathrm{N})$ & $\mathrm{pH}$ \\
\hline 'Sun 7705' & 89.0 & 3.1 & 5.4 & 8.4 & 3.7 & 0.27 & 0.34 & 0.46 \\
\hline Sun $7705-\mathrm{F}_{2}$ & 118.9 & 0.8 & 21.3 & 9.1 & 2.1 & 0.16 & 0.38 & 0.41 \\
\hline 'Loreto' & 354.4 & 3.5 & 4.1 & 0.8 & 0.6 & 0.29 & 0.61 & 0.35 \\
\hline 'Loreto- $\mathrm{F}_{2}$ & 131.7 & 1.8 & 7.2 & 2.1 & 0.9 & 0.26 & 0.08 & 0.12 \\
\hline 'Moctezuma' & 296.3 & 3.0 & 9.7 & 1.5 & 3.3 & 0.15 & 0.29 & 0.27 \\
\hline Moctezuma- $\mathrm{F}_{2}$ & 149.6 & 1.7 & 20.8 & 8.4 & 5.2 & 0.68 & 0.29 & 0.25 \\
\hline 'Cuauhtémoc' & 205.8 & 2.3 & 7.6 & 0.5 & 1.3 & 0.34 & 0.15 & 0.27 \\
\hline Cuauhtémoc- $\mathrm{F}_{2}$ & 159.1 & 2.6 & 9.4 & 4.7 & 1.7 & 0.07 & 0.16 & 0.23 \\
\hline 'Reserva' & 398.9 & 5.1 & 5.4 & 1.8 & 1.2 & 0.28 & 0.42 & 0.48 \\
\hline Reserva- $\mathrm{F}_{2}$ & 81.0 & 1.3 & 5.4 & 8.4 & 3.3 & 0.21 & 0.76 & 0.10 \\
\hline 'Espartaco' & 365.4 & 4.6 & 13.9 & 1.9 & 2.0 & 0.44 & 0.25 & 0.66 \\
\hline Espartaco- $\mathrm{F}_{2}$ & 165.3 & 2.2 & 19.1 & 5.6 & 3.4 & 0.36 & 0.32 & 0.73 \\
\hline 'Cid' & 155.5 & 1.7 & 8.1 & 1.1 & 1.6 & 0.22 & 0.50 & 0.48 \\
\hline Cid-F ${ }_{2}$ & 75.9 & 1.0 & 2.6 & 4.2 & 3.1 & 0.38 & 0.29 & 0.37 \\
\hline
\end{tabular}

cuyos precios son mayores de 50 centavos de dólar por semilla (Juárez et al., 2000). Para el consumidor, la calidad del tomate viene determinada por atributos como apariencia externa, sabor y características nutritivas (González et al., 2004), además de firmeza, SST, acidez y tamaño de fruto, de las cuales depende también el precio en el mercado.

Un componente importante del rendimiento es el número de frutos por planta. En este caso, en cinco de los siete híbridos ('Reserva', 'Espartaco', 'Sun 7705', 'Moctezuma' y 'Cuauhtémoc'), solo hubo una tendencia de que las generaciones $\mathrm{F}_{2}$ tuvieran un menor NTF (Cuadro 2), con diferencias que no fueron significativas. El comportamiento de la $\mathrm{F}_{2} v s . \mathrm{F}_{1}$ de esta variable en 'Loreto' es interesante, ya que aunque $\mathrm{F}_{2}$ y $\mathrm{F}_{1}$ fueron estadísticamente iguales, la $\mathrm{F}_{2}$ tendió a ser mejor porque numéricamente presentó un aumento de $14.5 \%$. De acuerdo con De Vicente y Tanksley (1993), durante la segregación en $\mathrm{F}_{2}$ se pueden presentar combinaciones genéticas que generan individuos que en promedio se comportan mejor que el híbrido comercial. Este resultado concuerda con lo aquí observado en 'Loreto', que además es apoyado por el hecho de que la segregación transgresiva es común en autógamas (Rieseberg et al., 1999).

El hecho de que el tomate es una especie autógama con reducida variabilidad genética (Park et al., 2004), podría explicar el que la segregación aquí encontrada no fuera grande, como también lo reportó Warnock (1991).

Para las características de calidad SST, FIR y pH, no hubo diferencias estadísticas entre las medias de los híbridos comerciales $\mathrm{F}_{1}$ y sus respectivas generaciones $\mathrm{F}_{2}$, lo cual indica que había una reducida variación genética para estas características en la constitución genética de las líneas que dieron origen a los híbridos evaluados, y consecuentemente en las generaciones tempranas de los híbridos entre ellas.

En general, se puede decir que los resultados obtenidos en este trabajo permitieron identificar al menos un genotipo que en la generación $\mathrm{F}_{2}$ posee rendimiento y calidad similares a la generación $\mathrm{F}_{1}$, lo cual está en concordancia con la hipótesis planteada.

Las desviaciones estándar son una medida de dispersión que informan de las distancias medias que tienen los datos con respecto a su media aritmética. Para el caso de la magnitud de la variación que hubo en los genotipos $\mathrm{F}_{1}$ y $\mathrm{F}_{2}$ (Cuadro 3), aquí se observó una tendencia general de que la variación a través de genotipos fue menor en $\mathrm{F}_{2}$ para variables como PTF, NTF, SST, y pH; mientras que para otro grupo de variables como PPF, LF y DF la tendencia fue al contrario, ya que en estos casos los genotipos dieron mayor variación en $\mathrm{F}_{2}$. En el caso de la variable firmeza, varios valores de $F_{1}$ fueron muy similares a sus $\mathrm{F}_{2}$, sin haber una tendencia clara a través de todos los genotipos, con respecto a la variación en alguna de las generaciones filiales.

Cabe señalar que en general la producción de fruto fue baja, atribuible a las bajas temperaturas invernales y a la falta de calefacción en el invernadero donde se hizo la evaluación. Aun así, es de esperar que resultados similares se 
obtengan en condiciones de temperaturas más favorables.

\section{CONCLUSIONES}

Los pequeños productores de tomate podrían utilizar semillas $\mathrm{F}_{2}$ del híbrido 'Loreto' para siembras comerciales, sin que ello represente una pérdida significativa del rendimiento ni de las características de calidad del fruto, lo que puede reducir costos de producción.

En cambio, los híbridos 'Sun 7705’ y 'Moctezuma' tuvieron la mayor depresión endogámica, por la reducción en el rendimiento de fruto y en sus componentes en la generación $\mathrm{F}_{2}$.

Hubo amplia variación genética entre las generaciones filiales $\mathrm{F}_{1}$ y $\mathrm{F}_{2}$ de los híbridos, lo cual se reflejó en una amplia segregación en la forma del fruto y en sus características cuantitativas.

Se podría obtener germoplasma sobresaliente de tomate para el mejoramiento genético en México a partir de híbridos $\mathrm{F}_{1}$ comerciales, ya que en la generación $\mathrm{F}_{2}$ es posible detectar individuos con rendimiento y cualidades similares a los de la generación $\mathrm{F}_{1}$.

\section{BIBLIOGRAFÍA}

Bai Y, P Lindhout (2007) Domestication and breeding of tomatoes: what have we gained and what can we gain in the future? Ann. Bot. 100:1085-1094.

Barrett H C, A M Rhodes (1976) A numerical taxonomic study of affinity relationships in cultivated Citrus and its close relatives. Syst. Bot. 1:105-136.

Bartkaite O (2001) Evaluation of Tomato Heterosis Expression. Scientific Works. Horticulture and Vegetable Growing. Lithuanian Institute of Horticulture. Babtai, Kaunas district, Lithuania. pp:1012.

Charlesworth D, B Charlesworth (1987) Inbreeding depression and its evolutionary consequences. Annu. Rev. Ecol. Syst. 18:237-268.

De Giglio M A (2003) Growth of the fresh greenhouse tomato market in the USA. Acta Hort. 611:91-92.

De Miranda P C, M L Anderson (2001) La complejidad de los materiales híbridos. USA. Seed News. Año XV N. 6. Disponible en: http://www.seednews.inf.br/_html/site_es/content/reportagem_capa/index.php?edicao $=60$ (Julio 2013).

De Vicente M C, S D Tanksley (1993) QTL analysis of transgressive segregation in an interespecific tomato cross. Genetics 134:585-589.
Escalona C V, V P Alvarado, M H Monardes, Z C Urbina, B A Martin (2009) Manual de Cultivo de Tomate (Lycopersicon esculentum Mill.). Disponible en: http://www.cepoc.uchile.cl/pdf/manua_ cultivo_tomate.pdf. (Junio 2012).

FAO, Food Agricultural Organization (2012) Organización de las Naciones Unidas para la Agricultura y la Alimentación. Disponible en: www.apps.fao.org. (Junio 2012).

Foolad R M (2007) Genome mapping and molecular breeding of tomato. Int. J. Plant Genomics 2007:1-52.

González S R F, M A Martínez D (2002) Dumping: El caso del jitomate, México 1996. Com. Socioecon. Estad. Inform. 6:1-30.

González C A, M del C Salas S, M Urrestarazu G (2004) Producción y calidad en el cultivo de tomate cherry. In: Tratado de Cultivos sin Suelo. M Urrestarazu Gavilán (ed). Ed. Mundi-Prensa. Madrid, España. pp:703-747.

Jarne P, D Charlesworth (1993) The evolution of the selfing rate in functionally hermaphrodite plants and animals. Ann. Rev. Ecol. Syst. 24:441-466.

Juárez L G F, F Sánchez del C, E Contreras M (2000) Efectos del manejo de esquejes sobre el rendimiento de jitomate (Lycopersicon esculentum Mill.) en hidroponía. Rev. Chapingo S. Hort. 6:19-23.

Martínez S J, A Peña L , J E Rodríguez P, C Villanueva V, J Sahagún C, M G Peña O (2005) Comportamiento productivo en híbridos de jitomate y sus respectivas poblaciones $\mathrm{F}_{2}$. Rev. Chapingo. S. Hort. 2:299-307.

Mohamed M F (1998) Characteristics and inheritance of natural facultative parthenocarpic fruit-set in 'Nadja' tomato under low temperature conditions. Euphytica 103:211-217.

Nieto A R, E Velasco H (2006) Cultivo de Jitomate en Hidroponía en Invernadero. 2a ed. Departamento de Fitotecnia, Universidad Autónoma de Chapingo. México. 130 p.

Park Y H, M A L West, D A St Clair (2004) Evolution of AFLPs for germplasm fingerprinting and assessment of genetic diversity in cultivars of tomato (Lycopersicon esculentum L.). Genome 47:510-518.

Poehlman J M, D S Allen (2003) Mejoramiento Genético de las Cosechas. Ed. Limusa. D.F., México. pp:172-176.

Rieseberg L H, M A Archer, R K Wayne (1999) Transgressive segregation, adaptation, and speciation. Heredity 83:363-372.

SAS Institute (2002) User's Guide of SAS (Statistical Analysis System). SAS Institute Inc. Cary, N. C. USA. 550 p.

Sahagún C J, F García M (2009) El coeficiente de endogamia de una población bajo selección masal. Agrociencia 43:119-132.

Scott J W (2008) Fresh market tomato breeding in the USA. Acta Hort. 789:21-26.

Shivaprasad P V, R M Dunn, B A C M Santos, A Bassett, D C Baulcombe (2012) Extraordinary transgressive phenotypes of hybrid tomato are influenced by epigenetics and small silencing RNAs. EMBO J. 31:257-266.

Steiner A A (1984) The universal nutrient solution. In: Proc. 6th International Congress on Soilless Culture. Wageningen, The Netherlands. pp:633-650.

Stevens M A (1973) The influence of multiple quality requirements on the plant breeding. HortScience 8:110-112.

Warnock S J (1991) Natural habitats of Lycopersicon species. HortScience 26:466-471. 